\title{
Cutaneous adverse effects of imatinib mesylate
}

\author{
Mehmet Hilmi Dogu, Ismail Sari, Sibel Hacioglu, Ali Keskin
}

Department of Hematology, Pamukkale University Faculty of Medicine, Denizli, Turkey

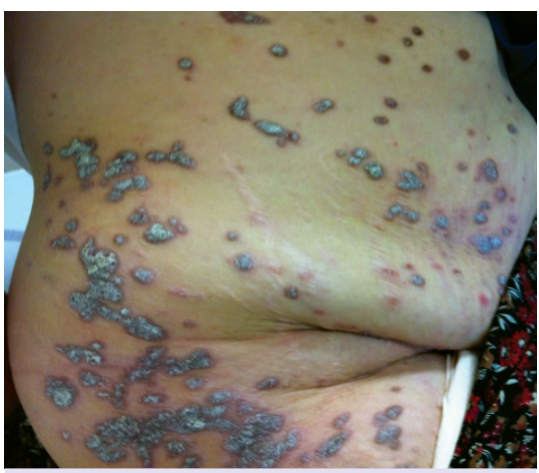

FIGURE1. Right lateral image of patient.

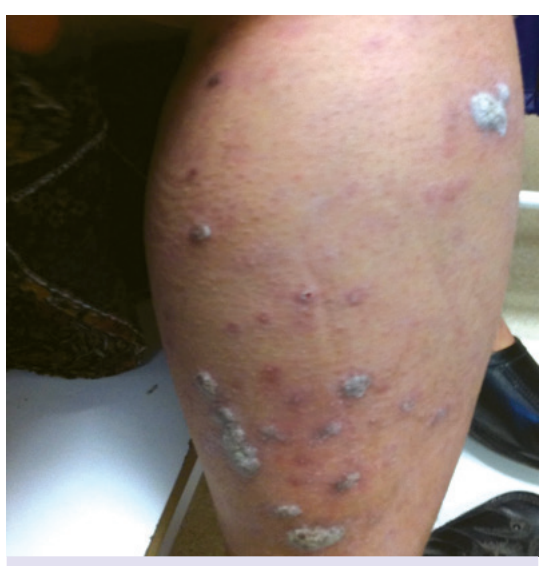

FIGURE2. Right leg image of patient.
A 52-year-old female patient was found to have elevated white blood cell count (WBC) on routine physical examination. Her WBC count was 67.260/ $\mathrm{uL}$, hemoglobin was $8.3 \mathrm{gm} / \mathrm{dL}$, hematocrit was $26.1 \%$ and platelet count was $133,000 / \mathrm{uL}$. Bone marrow reveled myeloid hyperplasia and the Philadelphia chromosome was positive. Patient was diagnosed as chronic myeloid leukemia (CML) and imatinib mesylate treatment began in August 2011. The patient did not receive any concomitant treatment. On the $5^{\text {th }}$ month of imatinib treatment, the patient was admitted because of pruritus and grade 4 generalized skin rashes. Erythematous lesions and scaly plaques were noted (Figure 1,2). At that time, imatinib was discontuined and topical corticosteroid was given with oral an antihistaminic. Her pruritus resolved and skin lesions improved. Imatinib was replaced by dasatinib $(100 \mathrm{mg} /$ day $)$ and her treatment is still ongoing. Chronic myeloid leukemia (CML) is a myeloproliferative neoplasm with an incidence of one-two cases per 100,000 adults and accounts for nearly $15 \%$ of newly diagnosed cases of leukemia in adults. CML is characterized by a balanced ge- netic translocation, $\mathrm{t}(9 ; 22)(\mathrm{q} 34 ; \mathrm{q} 11,2)$. This rearrangement is known as the Philadelphia chromosome. Imatinib mesylate was the first TKI to receive approval by the Food and Drug Administration (FDA) for the treatment of patients with CML-CP. Cutaneous reactions to imatinib are common. The reported frequency varies across studies (rash, 32\%-39\%, severe rash 3\%$4 \%$; pruritus, $6 \%-10 \%$, severe pruritus $<1 \%$; severe cutaneous reactions, $<1 \%$, and photosensitivity $0.1 \%-1 \%$ ). The median onset of severe skin reaction is about 1-2 months after the start of treatment but it may be delayed. Management of dermatologic adverse events is generally confined to discontinuation or reduction of imatinib dose, and administration of oral and/or topical corticosteroid and antihistamines use. In our patient due to the grade 4 skin reactions, molecular response has not been achieved yet. As we know, in clinical trials with $2^{\text {nd }}$ generation TKIs significantly deeper and faster responses have been reported, however their impact on long-term survival remains to be determined. In accordance with this clinical studies we replaced imatinib with dasatinib at daily doses of $100 \mathrm{mg}$. 